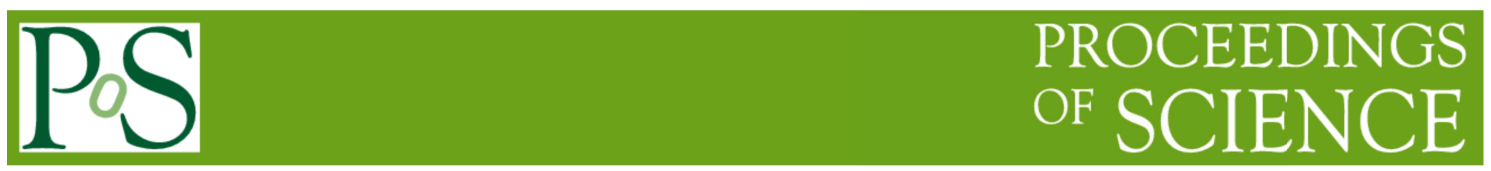

\title{
SALT Investigations of Transients
}

\author{
David A.H. Buckley ${ }^{1}$ \\ South African Astronomical Observatory \\ PO Box 9, Observatory 7935, Cape Town, South Africa
}

In this talk I present the case for why observing transient objects with SALT is a compelling and important science driver, particularly in the era of larger and more ambitious transient detection surveys, like the future LSST. SALT is well suited to undertake target of opportunity observations of transients in order to properly characterize and understand them. Examples of successful transient programs are listed and some results presented here, which include followup observations of optical transients from the recently installed MASTER-SAAO transient detection facility. This system is described, plus the results of the 143 transient discoveries made in the first $\sim 8$ months of operations, which include a variety of objects: Catalysmic Variables (mostly Dwarf Novae), Supernovae, Active Galactic Nuclei (particularly blazars), flare stars, long period binaries and Gamma Ray Bursts. I finally discuss some of the new facilities at SAAO which will come online in 2016 (MeerLICHT, MONET-South and a new robotic $1 \mathrm{~m}$ telescope), which will be important additions to the suite of telescopes and instruments able to conduct observations of transients and targets of opportunity. Together with SALT and other multi-wavelength facilities (including local facilities like HESS II and MeerKAT and satellites like Fermi, Swift and ASTROSAT), the future looks promising for exciting new transient science programs with these facilities working together.

SALT Science Conference 2015 -SSC2015-

1-5 June, 2015

Stellenbosch Institute of Advanced Study, South Africa

${ }^{1}$ Speaker 


\section{Introduction}

Observational studies of transient phenomena in the Universe is becoming a growth industry, particularly with the advent of faster, wider and deeper panchromatic imaging surveys of the sky, both with ground and satellite based observatories. There is a growing interest amongst astronomers in conducting multi-wavelength followup studies of astrophysical transients, and programs supporting such studies have already been initiated using SALT and other complementary facilities. Programs conducted on SALT have already included followup studies of high energy events, like X-ray and $\gamma$-ray transients, gamma ray bursts (GRBs), and blazars, and will continue into the future. The same is the case for studies of transients detected at other wavelengths (e.g. optical or IR), including supernovae, cataclysmic variables, novae and other eruptive variables. Most of these alerts have come from the likes of ground-based detection systems, like PTF [1], ASAS-SN [2], CRTS [3] and MASTER [4], while the significance of space-based systems, like GAIA and Spitzer, is becoming increasingly important (e.g. see [5]). When the MeerKAT radio telescope array begins its science operations (2016/17), there will be a dedicated optical telescope at Sutherland, MeerLICHT [6], which will simultaneously monitor MeerKAT fields in order to detect and observe optical counterparts of MeerKAT detected radio transients.

For prompt optical identification and followup of transients, it is often imperative to observe such events as close to the initial alert time as possible (e.g. $<1000-2000 \mathrm{~s}$ for GRBs). Until recently (Dec 2014), all transient alerts followed up in South Africa (e.g. at SALT) came from ground-based facilities on other continents, or from satellite missions. This has meant that many followup observations could often only happen $\sim 24$ hours after the initial alert. This has now changed with the establishment at the SAAO observing station in Sutherland of the first local optical transient detection system, namely MASTER-SAAO, which will be described later.

With the recent development of new dedicated and larger scale survey facilities (e.g. PanSTARRs [7] and SkyMapper [8]) and those in the future (e.g. LSST [9]), the opportunities to study the transient Universe will become unprecedented. Indeed the volume of alerts (up to $\sim 10^{6}$ per night) will mean that the traditional manner of conducting followup programs will require automated responses to classify transients and to decide which are worthy of more detailed followup observations. New techniques, for example employing machine learning, will need to be employed in order to optimally process the shear volume of data. The experiences gained with existing facilities (like MASTER-SAAO) will aid us in the task of how to conduct larger scale programs in the future, utilizing facilities like the robotic telescopes soon to come on-line in Sutherland (e.g. MONET-South and the new SAAO 1-m robotic telescope), and those that might come in the future (e.g. a 4-m class telescope).

In this paper I firstly review some of the issues associated with observing transients with SALT, and present some examples of successful observations carried out and plans for future programs. The MASTER-SAAO system and future facilities will be reviewed and some preliminary results of followup studies on transients discovered by the former will be presented, including observations from SALT Director's Discretionary Time (DDT). 


\section{Observing with SALT}

The Southern African Large Telescope (SALT) has proven itself to be an excellent tool for the study of astrophysical transients and targets of opportunity (ToO), mainly because of its queue scheduled operation. This, coupled with the flexibility in choice of instrumentation, where it takes only a minute or so to change between instruments, makes it ideally suited to followup of transients detected in real time. The major issue in conducting SALT transient followup observations is target availability and the timescale over which such observations can begin to be attempted.

Because of SALT's design, namely the fixed altitude angle of the telescope structure, the instantaneous viewing window for SALT is a $12^{\circ}$ wide annulus offset from the zenith by $37^{\circ}$. Thus only $\sim 12 \%$ of the visible sky is available to SALT at any given time, which implies that observations have to be carefully planned for when the object of interest is accessible to SALT. The instantaneous viewing window for SALT is shown in Figure 1 in terms of an hour angle vs. declination plot. Only sources inside the blue annulus (referred to by the SALT Astronomy Operations team as the SALT "toilet seat" diagram) can be observed at any given time and the length of the observing window is simply the extent of an appropriate horizontal line within the annulus at a give constant declination. Thus conducting prompt followup observations (e.g. < few $1000 \mathrm{~s}$ from an alert), like GRBs, poses a challenge, since often the object is only accessible on much larger timescale, like hours. Nonetheless, several successful ToO programs involving transients have been conducted with SALT, particularly where the required timescale for followup $>24 \mathrm{~h}$, and I would argue that this has become a major competitive advantage of the facility.

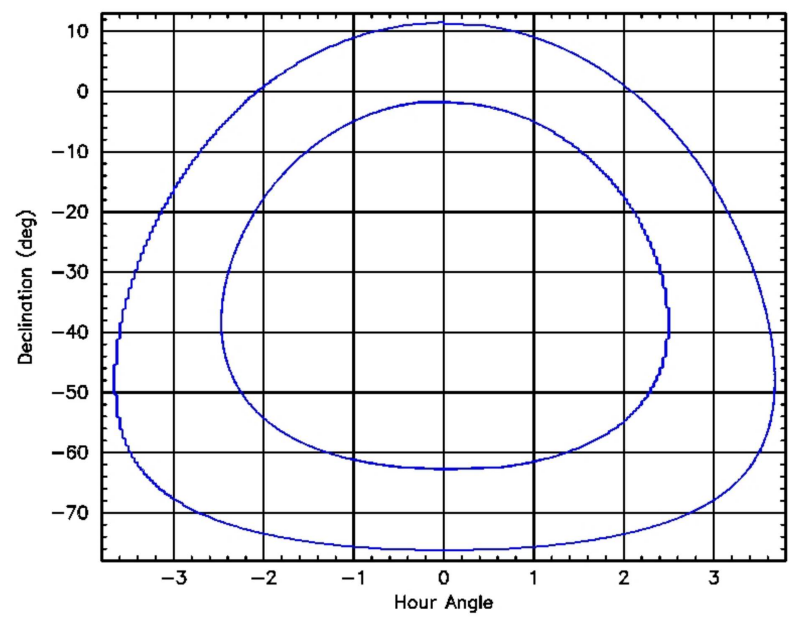

Figure 1: The instantaneous SALT viewing window (SALT "toilet seat"). Only objects within the annulus are observable at any given instance.

In keeping with the theme of this conference, the science productivity of SALT and its increasing standing and reputation as a global facility can only be enhanced by focusing on those areas for which SALT has a competitive advantage. Rapid followup observations of transients and ToOs is one of those areas, because of the following reasons: 
- SALT's $100 \%$ queue scheduled service modus operandi [10]

- The wide variety of available instruments/modes [11]

- Rapid instrument changes and mode reconfigurations

- Flexible scheduling allowing for synoptic monitoring at difference cadences

- High time resolution capabilities

- Ability to observe Targets of Opportunity can be done at short notice

It is also worth emphasizing that it is to the SALT community's benefit that only appropriate programs are considered for SALT and to utilize it in the best possible way to maximize the chances of success (e.g. see [12] in these proceedings).

In defining appropriate SALT programs, cognizance of the following points should be taken:

1. SALT is potentially a $7-8 \mathrm{~m}$ class telescope (based on effective area), but due to current issues (e.g. lack of a working active optics control system) it is performing more like a 5-6-m class telescope

2. Even when the full active optics system is finally working (hopefully in 2016), SALT will never have the imaging ability of similar aperture class telescopes, like Keck, VLT, Gemini or Subaru. This is due to the two reasons listed next:

3. The Sutherland site has poorer seeing than other 8 to $10-\mathrm{m}$ telescope sites, with a median seeing (measured at $\sim 1.5$-m above ground level at the zenith) of $\sim 1.2$ arcsec.

4. There is no Adaptive Optics system on SALT

5. SALT's restrictive viewing window, which limits the available time on a given field

6. Avoidance of over-subscription of certain fields (e.g. SDSS fields)

7. Current issues with accurate flat fielding and photometric calibrations due to complications with the optical design and moving pupil

8. restrictions on the guidance system performance and efficiency, particularly for MOS modes, where there is currently no closed-loop field rotation correction, which limits exposure times

9. Difficulty in co-adding many observations to reach fainter limits due to systematic errors (e.g. due to point 7.)

To maximize the chances of successfully completing scientifically compelling programs, SALT programs should, where possible, try to limit restrictions by adopting the following practices:

- Request realistic seeing constraints, ideally with modest seeing limits (e.g. $\sim 2 \operatorname{arcsec}$ ) if possible

- Keep observing blocks short, ideally $0.5-1 \mathrm{~h}$. The shorter blocks allow for more flexibility for scheduling, though the trade-off is less efficiency, due to the increasing \% of overheads. But it often comes down to whether the block will even be attempted, despite its efficiency, particularly for over-subscribed Priority 3 blocks competing with higher priority blocks.

All the news is not bad and SALT does have a competitive edge in many areas: 
- The ability to observe faint diffuse emission (e.g. Fabry-Perot observations)

- The ability to conduct high time resolution observations (photometry and spectroscopy)

- Certain instrument modes are unique or rare, for example:

- Fabry-Pérot modes

$\circ \quad$ polarimetric modes

- very high time resolution modes

- Synoptic monitoring on a variety of timescales (days, weeks, months, semesters)

- $\quad$ and, the main point of this paper, the ability to react quickly to ToOs and transients

\section{Observing transients and targets of opportunity with SALT}

As SALT is a $100 \%$ queue scheduled telescope, in principle it is a lot easier to arrange target of opportunity (ToO) followup observations of transients than with conventionally scheduled telescopes, notwithstanding the previous comments regarding the restrictive viewing window. The instrumentation available on SALT is well suited to a variety of followup observations of transients, particularly spectroscopy for the characterization of the object and time resolved studies. The four science instruments on SALT [12] currently comprise of:

- SALTICAM, a broad-band UV-visible imaging camera, with high time resolution

- RSS, the Robert Stobie Spectrograph, a versatile prime focus instrument

- SALT HRS, a stable fibre fed high resolution échelle spectrograph

- $\quad$ BVIT, the Berkeley Visible Image Tube photon counting camera (a visitor instrument)

Table 1 outlines the potential types of transient followup observations that can be undertaken with SALT and the respective instruments and observing modes which could be utilized. The faintness limits for such observations are very dependent on observing conditions and the quality of the primary mirror alignment (in the absence of active mirror control, to be addressed in 2016).

Table 1: SALT Instrument Capabilities for Transient Followup

\begin{tabular}{|l|l|l|l|}
\hline Type of Observations & Instrument & Mode & Types of Objects \\
\hline Classification spectroscopy & RSS & Longslit; R $~ 350-1000$ & SNe; XRTs; GRBs; CVs; AGN \\
\hline Spectral line diagnostics & RSS & Longslit; R $~ 3000-6200$ & CVs; AGN \\
\hline & HRS & R 16,000 - 80,000 & V < 16; bright em. line sources \\
\hline Radial velocities & RSS & Longslit; R > 2000 & binaries \\
\hline & HRS & R 17,000 - 80,000 & V < 14; exoplanets \\
\hline Time res. Spectroscopy & RSS & Frame Transfer/Slot & 2 s/0.1 s resolution; CVs; XRTs \\
\hline Spectropolarimetry & RSS & Linear, circular, all-Stokes & GRBs; SNe; CVs; AGN \\
\hline Imaging polarimetry & RSS & Linear, circular, all-Stokes & GRBs; SNe; CVs; AGN \\
\hline High speed photometry & SALTICAM & Frame transfer/Slot & 2 s/0.06 s resolution; CVs; XRTs \\
\hline & BVIT & Photon counting & 50 ns time tagging; CVs; XRTs \\
\hline
\end{tabular}

"mode currently being re-commissioned (Oct 2015) 


\section{Examples of results of SALT transient ToO followups}

A number of SALT programs have been devoted to the observations of transients and Targets of Opportunity. They have covered a variety of topics which have resulted in a number of publications, with more in progress. In Table 2 I list some of these SALT programs, and two examples of some spectra are shown in the following figures (Figure 2 and 3). Apart from dedicated ToO or transient followup programs approved by the SALT TACs, DDT has also been used for short one-off observations of compelling transients. However, currently only $\sim 10 \mathrm{~h} \mathrm{a}$ semester is available for potential DDT programs.

Table 2: Examples of SALT Transient/ToO Followup Programs

\begin{tabular}{|l|l|l|}
\hline Transient Type & Nature of Progam & PI / SALT Partner \\
\hline Supernovae & Rapid classification of new SNe & Fesen / Dartmouth \\
\hline & Observations of new \& late time SN Ia & Jha / Rutgers \\
\hline GRBs & Observing SN Ia from Dark Energy Survey & Kasai / UCT \\
\hline Asteroids & Afterglow spectroscopy of GRBs & Buckley / SAAO \\
\hline Planets & Detecting NEOs & Kwiatowski / Poland \\
\hline X-ray transients & Mutual events of Neptunian satellites & Christou / UKSC \\
\hline Super Soft Sources & Observations of transient SSSs & Kotze / SAAO \\
\hline mCVs & Followup spectroscopy of CRTS sources & Charles / SAAO, UKSC \\
\hline Novae & Spectroscopy of new Novae & Woudt / UCT (DDT) \\
\hline AGN outbursts & Spectroscopy & Kollatschny / Göttingen (DDT) \\
\hline HMXB outburst & Spectroscopy (+ KAT observations) & Schurch / UCT \\
\hline Be-XRB outburst & Spectroscopy of a SMC X-ray pulsar & Bartlett / UCT (DDT) \\
\hline MASTER alerts & Spectroscopy of new CV and blazar transients & Kniazev / SAAO (DDT) \\
\hline
\end{tabular}

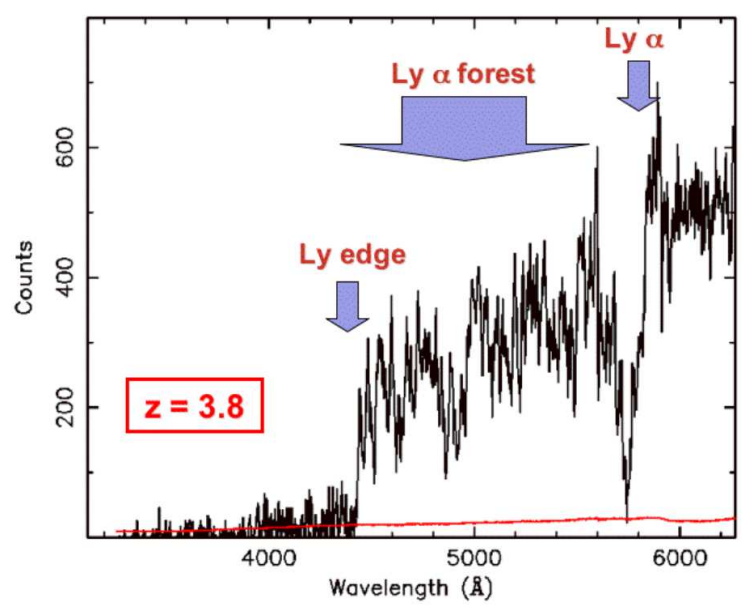

Figure 2: The first GRB spectrum obtained with SALT, of GRB 060605, identified as a Lyman break galaxy at $\mathrm{z}=3.8$. The GRB had faded to $\mathrm{V} \sim 21$ at the time of the observation, which was $8 \mathrm{~h}$ after the initial Swift alert [13]. 


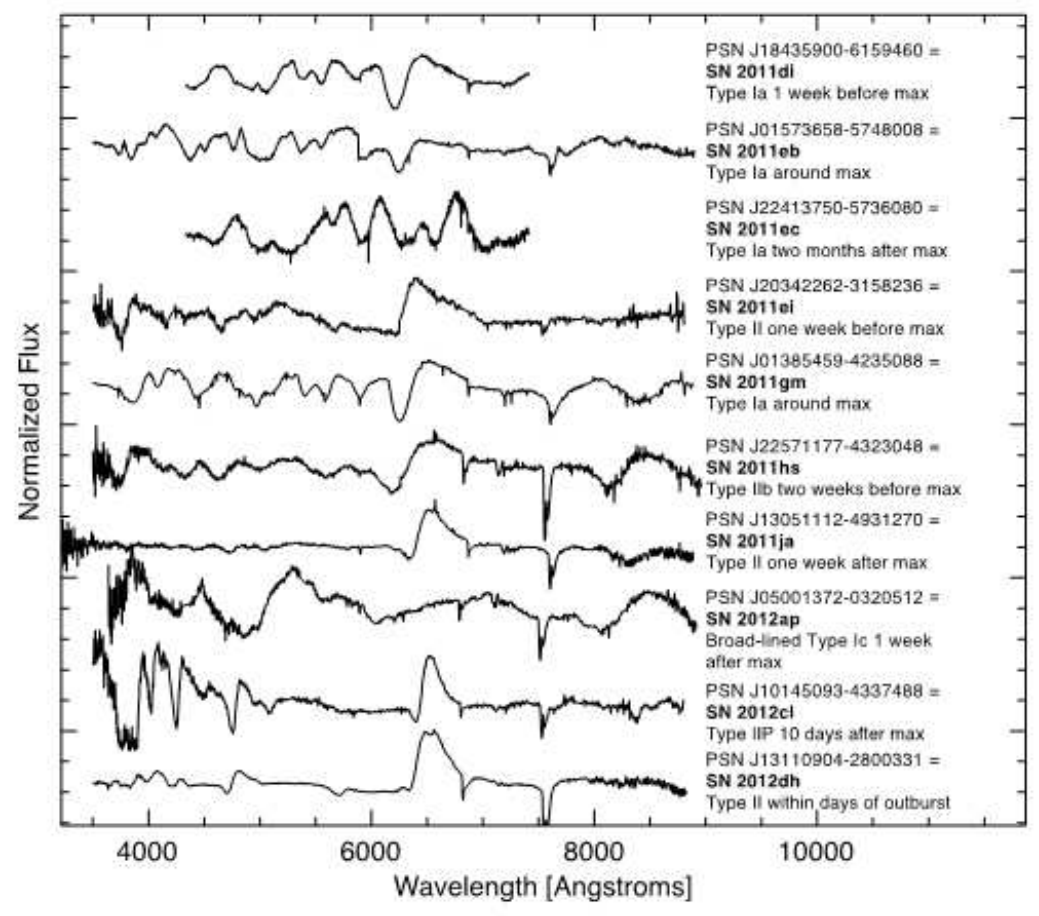

Figure 3: Examples of Supernova spectra taken with SALT as part of a ToO followup program of new $\mathrm{SNe}$ candidates[14]

Some of the results from these programs are illustrated in Figures 2 and 3, where I show observations of the one successful SALT GRB observation to date, GRB 060605 [13], and SALT spectra of new supernova discoveries [14].

Since MASTER-SAAO has been operating (see next section), 143 optical transient discoveries have been made. Although it was planned to undertake classification spectroscopy with SALT RSS of a subset of some of the more compelling transient detections, a proposal to do this in semester 2015-1 (from 1 May 2015) was unsuccessful, so the only observations done to date (of three transients) have been through an allocation of $\sim 6700 \mathrm{~s}$ of SALT DDT. From 1 Nov 2015, an approved program of followup SALT spectroscopy will begin for two semesters (until 31 Oct 2016).

The three MASTER-SAAO transients which were observed by SALT include two new CVs plus one flaring blazar. Most of the MASTER-SAAO transient detections ( 77\%) are Cataclysmic Variables of the Dwarf Nova (DNe) sub-class, which undergo recurrent optical outbursts due to accretion disc instability cycles, recurring on timescales of weeks to years. These new discoveries have outburst amplitudes in the range $2^{\mathrm{m}}<\Delta \mathrm{m}<7^{\mathrm{m}}$. The higher amplitude DNe are lower luminosity (so fainter), short orbital period $(<2 \mathrm{~h})$, with the WZ Sge subclass being the faintest and with the highest outburst amplitudes, lowest accretion rates and shortest orbital periods of all of them. It is this group which are potentially interesting for followup studies, since they could represent a "hidden iceberg" population, important for understanding CV evolution.

One such DNe observed by SALT was MASTER_OT_J074858.45-655344.6, a $B \sim 17^{\mathrm{m}}$ magnitude transient with a $\sim 3^{\mathrm{m}}$ amplitude discovered serendipitously during a search for the 
optical counterpart of the Gamma Ray Burst, GRB150114, discovered by Fermi. The SALT spectrum was typical of a DNe declining from outburst [15]. The second CV observed by SALT was first discovered to be an eclipsing synchronized magnetic CV (a Polar) through optical photometry with the SAAO 1.9-m telescope, immedaitely following its discovery. It showed the typical spectrum of a Polar, with strong and broad HeII Å4686 emission [16] (see Figure 4).

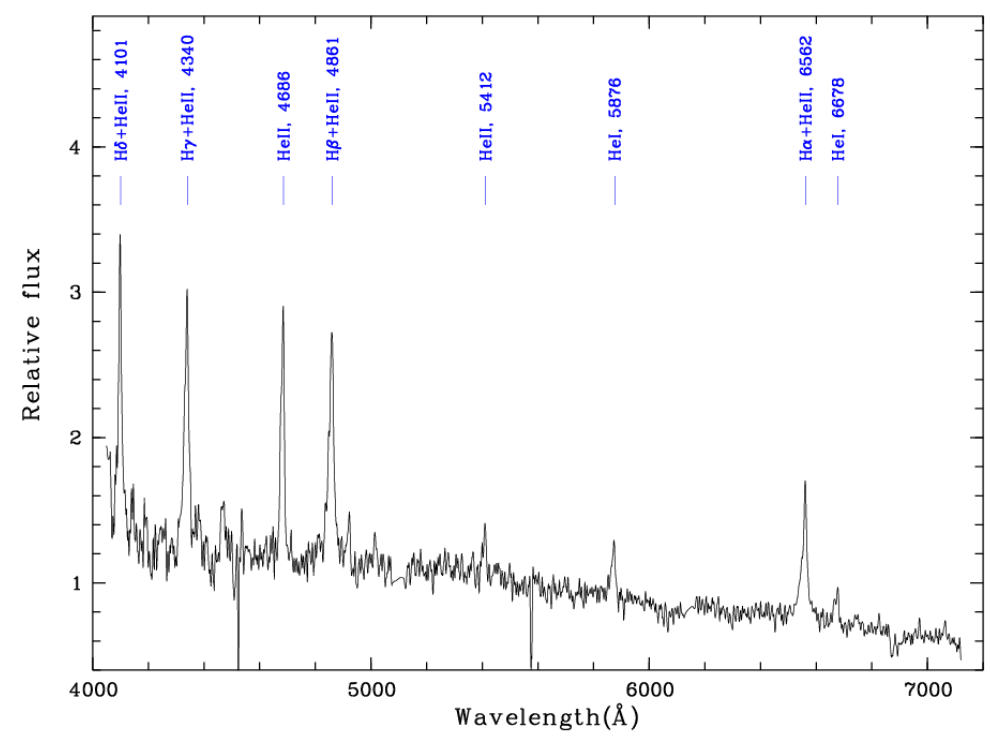

Figure 4: A SALT RSS spectrum (800 s exposure) of the newly discovered eclipsing polar, MASTER OT J061451.70-272535.5 [16].

The third MASTER transient observed by SALT was a flaring blazar, NVSS_J141922.55083832.0, detected in the $2^{\text {nd }}$ Fermi catalogue (2FGL J1419.4-0835), which brightened by $\sim 4^{\mathrm{m}}$ in Feb 2014. The MASTER alert also triggered X-ray observations with Swift. The possible detection of the Mg II $2798 \AA$ line at $\sim 5325 \AA$ in the SALT spectrum (Figure 5) has led to a redshift estimate of $z=0.90$ [17].

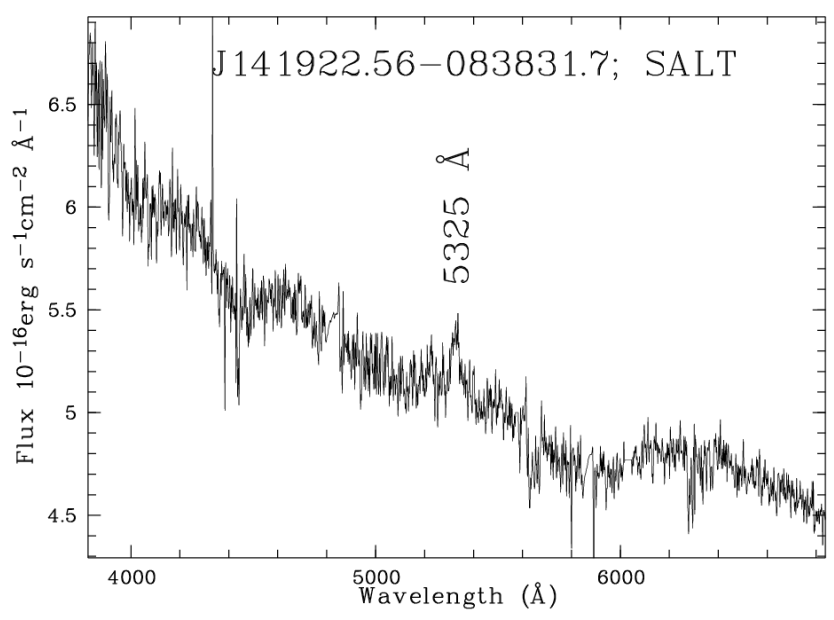

Figure 5: A SALT RSS spectrum of the flaring blazar, NVSS_J141922.55-083832.0 (= 2FGL J1419.40835), with a tentatively identified MgII emission line, implying $z=0.90$ [17]. 
This blazar was further observed by Fermi and monitored by MASTER, which measured $\sim 10 \%$ linear polarization. Historical data have shown that another optical flare occurred in Oct 2014, also detected by Fermi. The SEDs which have been derived from the X-ray (Swift \& Fermi), UV (Swift), optical (SAAO/ SALT), near IR (Guillermo Haro Observatory) and radio (GMRT) are currently under analysis and will feature in a future paper.

These initial MASTER transient results (currently being written up in two refereed papers), from a mere $\sim 6700 \mathrm{~s}$ of observations, have demonstrated the productivity of SALT transient followup observations. With the recent allocation of a more sizable amount of time in subsequent semesters $(\sim 60,000 \mathrm{~s}$ in total), it is expected that the result from these observations will be equally fruitful.

\section{The MASTER-SAAO facility: establishment and initial results}

The MASTER-SAAO optical transient detection system was installed at Sutherland in Dec 2014 (see Figure 6), and is part of a network of six identical MASTER II telescopes [4, 18], four in Russia and one recently established (May 2015) on the Canary Islands. An additional smaller facility, with a very wide field camera, in Argentina, is the only other MASTER node located in the southern hemisphere, at present. The six MASTER II facilities consist of identical dual 40 $\mathrm{cm}$ telescopes on a common equatorial mount, employing a wide field catadioptric Hamilton design [18] and using prime focus Apogee Alta U16M cameras. The telescope tubes can either be co-aligned, each surveying an identical $2^{\circ} \times 2^{\circ}$ field, with two different filters or polarizers, or they can be misaligned to allow twice the sky coverage $\left(2^{\circ} \times 4^{\circ}\right)$ using identical filters (or no filters at all, in the case for survey mode). The latter is the default mode for detecting optical transients, for which each MASTER telescope surveys up to $400-500$ degrees $^{2}$ per night. Each MASTER field is observed with three repeat $60-180 \mathrm{~s}$ exposures (dependent on Moon phase), which can reach a typical limiting instrumental magnitude of $19^{\mathrm{m}} .8$ in dark Moon conditions, or a magnitude or so less in brighter Moon. Co-adding multiple frames, as is done in followup mode (e.g. for GRB afterglow observations), has reached a limit of $22^{\mathrm{m}}$.3. MASTERSAAO has a declination range $+40^{\circ}>\operatorname{Dec}>-90^{\circ}$, though the survey is focused on southern fields, including the Galactic plane, but with less frequent coverage there. The typical cadence for a major survey field (south and out of the plane) is 7 - 10 days.

All MASTER optical transient discoveries are listed on the MASTER website (http://observe.pereplt.ru/MASTER OT.html) and are immediately published as Astronomer's Telegrams (ATels). Any results pertaining to GRB detections are also published in GCN circulars. The MASTER website includes discovery and reference images, coordinates, magnitudes and outburst amplitudes, where there is an existing pre-alert image. MASTER is one of the most successful transient detection systems, as evidenced by the fact that up to 2014 it has been responsible for $\sim 25 \%$ of all ATel transient alerts, second only to Swift and the most of any ground-based optical alerts (CRTS is next with $\sim 7 \%$ ). A total of $~ 900$ transient discoveries have been made to date (i.e. by early Sep 2015) by the MASTER network over the last $\sim 4$ years. 


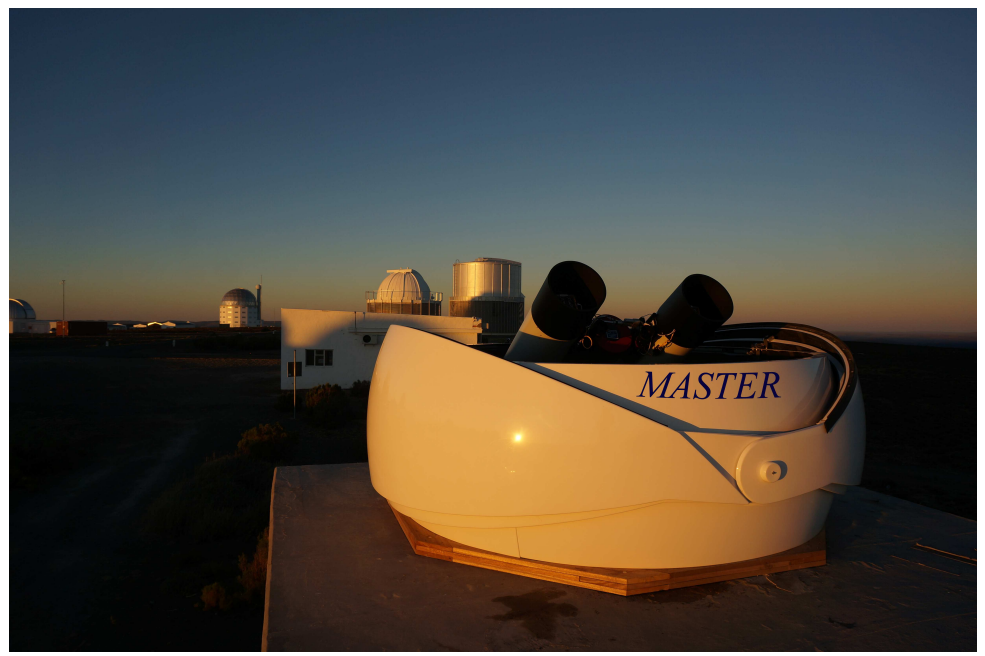

Figure 6: The robotic MASTER-SAAO facility at Sutherland, installed in December 2014. SALT can be seen on the horizon.

In the $\sim 8$ month period since MASTER-SAAO has been operational (since late Dec 2014), many southern hemisphere optical transients have been detected, while followup optical observations have also been undertaken by MASTER-SAAO of transient alerts from other sources, particularly GRBs and blazar outbursts. In Table 3 I list the breakdown of the 143 MASTER-SAAO optical transients discovered up to 9 Sep 2015, in terms of object class.

Table 3: Optical Transient Discoveries by MASTER-SAAO (16 Dec 2014 - 9 Sep 2015)

\begin{tabular}{|c|c|c|c|}
\hline Type & Class & Number & Comments \\
\hline \multirow{6}{*}{ Galactic objects } & Dwarf Novae CV in outburst & 109 & Criteria is $\Delta \mathrm{m}>2.2^{\mathrm{m}}$ \\
\hline & Novalike CV & 1 & \\
\hline & Magnetic CV (polar) & 1 & Eclipsing. Orb $\mathrm{P}=2.2 \mathrm{~h}$ \\
\hline & Flare Stars & 3 & \\
\hline & Long Period variables & 4 & \\
\hline & Anti-transient & 1 & Long period eclipser \\
\hline \multirow[t]{2}{*}{ Extragalactic } & Supernovae & 20 & \\
\hline & Gamma Ray Bursters & 2 & Detection from Swift alerts \\
\hline \multirow[t]{2}{*}{ Solar System } & Comets & 2 & First MASTER discoveries \\
\hline & Asteroids & Several & To be confirmed \\
\hline
\end{tabular}

In addition to the transient discoveries following from the MASTER-SAAO survey, a number of dedicated followup observations were also conducted with MASTER-SAAO, including of several GRB alerts (only 2 resulted in optical counterpart identifications) and blazar outburst alerts (7 observed). The two GRBs for which optical afterglows light curves were obtained by MASTER-SAAO were GRB141225A [19] and GRB 150301B [20]. These afterglows were detected at $113 \mathrm{~s}$ and $79 \mathrm{~s}$, respectively, after the Fermi and Swift triggers, at magnitudes of 17.5 and 15.3 , respectively. In the case of the latter, a unique prompt $\sim 8 \%$ linear polarization signal was measured in the early phases of the decline [21]. 


\section{Future transient observation plans at SAAO}

Many transient discoveries require followup observations to confirm their nature and to allow for more probing astrophysical observations. While there have already been several examples of followup observations (e.g. by SALT and other SAAO telescopes), some presented in the previous section, further plans are underway to expand the nature of these followup investigations. These include more comprehensive observations of the whole variety of optical transients currently being detected by alert networks, including GRBs, AGN, CVs and infrared, $\mathrm{X}$-ray and $\gamma$-ray transients. Optical followup observations with SALT and other SAAO telescopes will be supplemented, where appropriate, by other multi-wavelength facilities, including HESS II, ASTROSAT (the new Indian optical/UV/X-ray satellite) and eventually MeerKAT, once it begins operating. In addition, new optical followup possibilities at SAAO will be enabled with the establishment of two new robotic facilities at the SAAO, namely MONET-South and a new $1.0 \mathrm{~m}$ Robotic telescope.

While MASTER-SAAO is the first local optical transient detection system to be installed at the Sutherland station of the SAAO, it will be joined in 2016 by another transient detection facility, namely MeerLICHT [6]. This will be a $0.65 \mathrm{~m}$ telescope with a 2 degree $^{2}$ field of view, which will simultaneously observe fields being observed by the MeerKAT radio telescope. Another addition to the Sutherland suite of telescopes expected in 2016 is a new rapid-slewing alt-azimuth robotic 1-m telescope, which is currently under construction. A schematic of MeerLICHT and a photograph of a 1-m robotic telescope, similar to the one being procured for SAAO, are both shown in Figure 7 below.
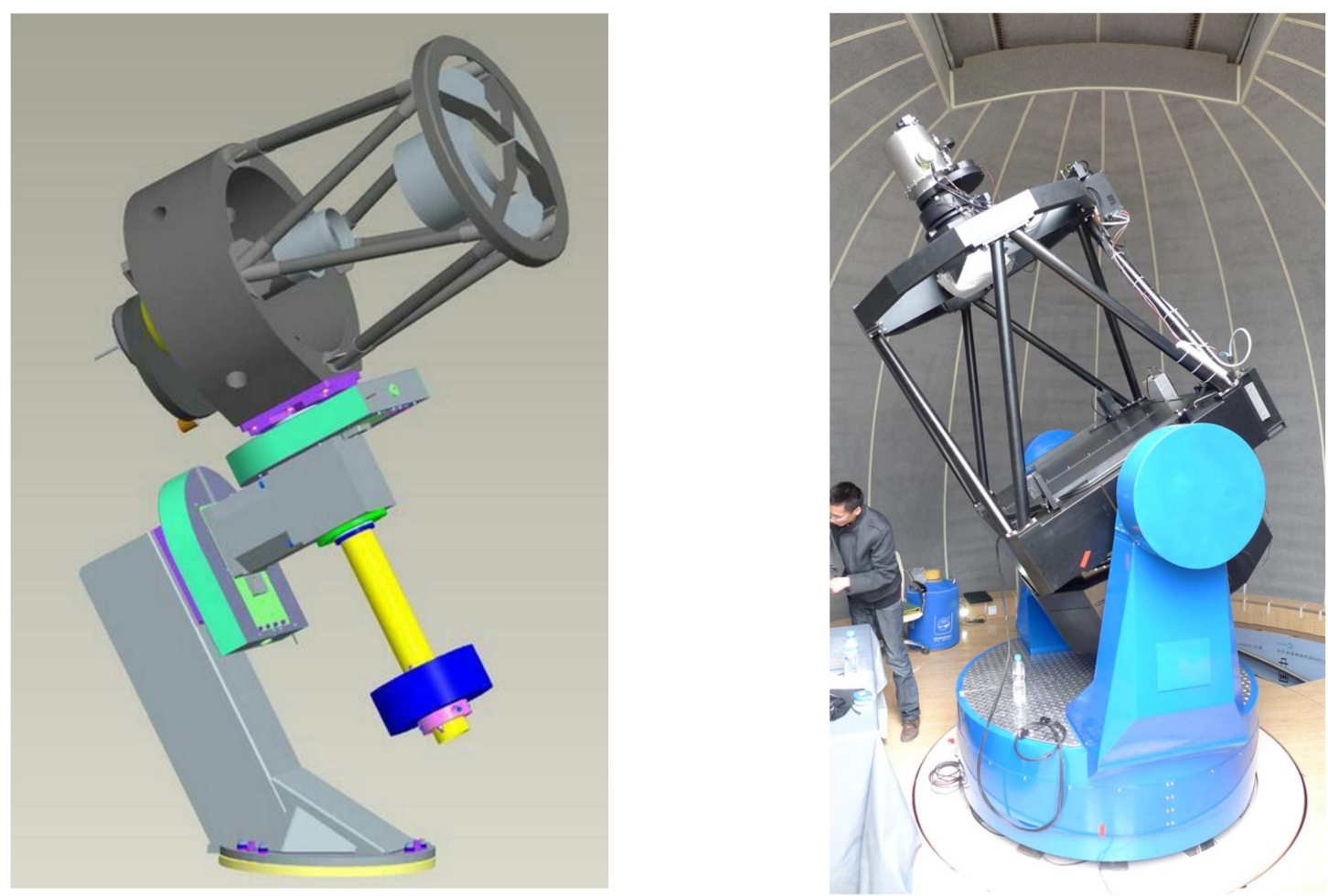

Figure 7. Left: schematic of the $0.65 \mathrm{~m}$ MeerLICHT telescope. Right: a similar telescope to the new SAAO $1 \mathrm{~m}$ robotic telescope. Both are due to be installed in Sutherland in 2016. 
MONET-South, owned by the University of Göttingen in Germany (see https://monet.unigoettingen.de/), is the twin telescope to MONET-North, situated at McDonald Observatory in Texas, USA. Both are robotic/remotely operated $1.2 \mathrm{~m}$ telescopes, with a major science goal being the followup of transients and ToOs. MONET-North has been operating for several years and MONET-South at Sutherland is expected to begin science operations shortly, initially with an imaging camera. A project has currently begun to design and construct an efficient fibre fed low resolution spectrograph (MORISOT), based on Darragh O'Donoghue's innovative STGS spectrograph design. With such an instrument, MONET-South will have the capability to rapidly spectroscopically classify many brighter $\left(V<\sim 16^{\mathrm{m}}-17^{\mathrm{m}}\right)$ optical transients as well as supporting coordinated multi-wavelength ToO observations.

While the final instrument suite is still to be determined for the new SAAO Robotic $1.0 \mathrm{~m}$ telescope, it will at least initially consist of an imaging camera and a high speed EM-CCD camera. Eventually it is planned to add fibre-fed low and/or high resolution spectrographs. The science goals for this telescope include synoptic monitoring of variable objects (stars and AGN), at a variety of cadences, but also supporting followup observations of transients and ToO.

Over the next year Sutherland will see the completion of two new facilities (SAAO Robotic $1 \mathrm{~m}$ telescope and MeerLICHT) and the commissioning of a third, namely MONETSouth. Together with MASTER-SAAO, there will then be two local transient detection facilities and two robotic followup telescopes. This will greatly enhance the ability to conduct transient science and coupled with the opportunities presented by SALT, a new era in transient astrophysical studies will be possible.

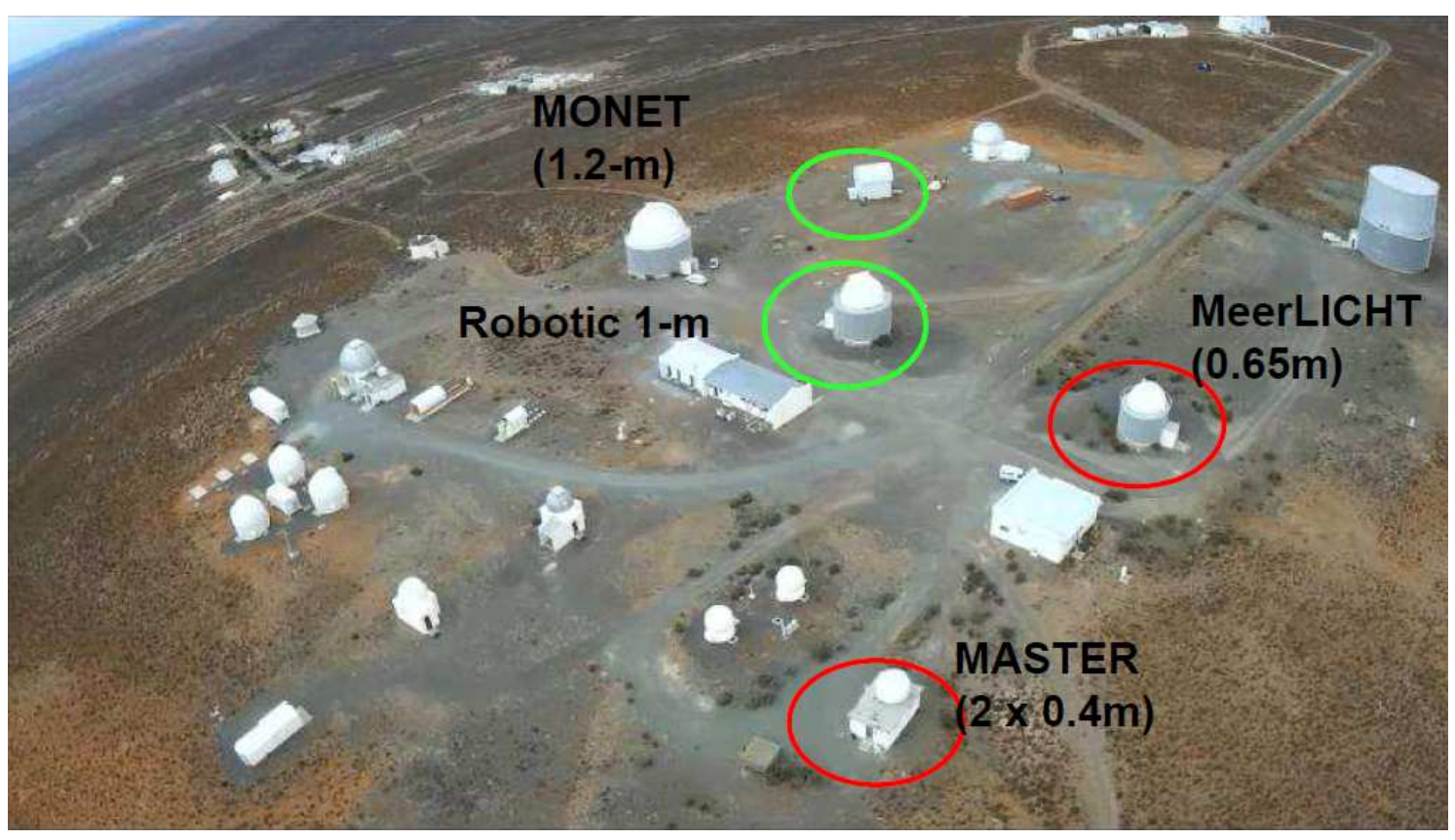

Figure 8: New transient detection (MASTER-SAAO and MeerLICHT) and followup (MONET and Robotic 1-m) facilities at Sutherland, the latter three which will become operational during 2016. 


\section{Summary}

I have presented results of transient and ToO followup studies that have utilized SALT and presented the case for why such programs are competitive. SALT's design and operation lends itself to such studies, which will likely become more important going into the future with the need for followup of large scale surveys, like LSST. The new optical transient detection facility at Sutherland, MASTER-SAAO, has been described and the first results from the transient survey and followup observations presented. Specifically, the results of 3 followup spectroscopic observations with SALT, on two CVs and a flaring blazar, were reported, and more detailed papers are currently in preparation. Finally I discussed the future possibilities of more expansive programs on transient followup observations at SAAO, exploiting the two new transient alert facilities (MASTER-SAAO and MeerLICHT) and two new robotic facilities (MONET-South and the SAAO $1 \mathrm{~m}$ Robotic Telescope) which will become operational in 2016. The experiences gained using SALT and these new SAAO facilities in the pursuit of understanding transient astrophysical phenomena will help to inform on how to conduct more ambitious larger scale transient followup campaigns in the future, for example, when LSST becomes operational.

\section{Acknowledgements}

I wish to acknowledge my collaborators and colleagues who have been involved in supporting transient science programs at SAAO and SALT in various ways, including observational support, modelling and establishing the MASTER-SAAO facility. These include Markus Böttcher, Hannes Breytenbach, Richard Britto, Sunil Chandra, Marissa Kotze, Alexei Kniazev, Mokhine Motsaoledi, Stephen Potter, Soeb Razzaque, K.P. Singh, C.S. Stalin, John Thorstensen and Patrick Woudt. I am also grateful for the support from the SAAO technical staff, particularly John Stoffels, Hilton Kamfer, Chantal Fourie and Piet Fourie, and also to the SAAO Director, T. Williams, for his support in establishing MASTER-SAAO. Also, thanks to Vladimir Lipunov for the initial suggestion of siting a MASTER telescope at Sutherland, plus his and his teams support of the MASTER program. As always, the support from the SALT operations staff in obtaining SALT data is much appreciated. These programs of transient detection and followup are also supported through the SA-GAMMA group and the National Research Foundation of South Africa, through research support grants to DB and others. Many of the observations discussed here were carried out on the Southern African Large telescope (SALT), some through an allocation of Directors Discretionary Time, for which I am most grateful.

Finally, but importantly, I personally wish to achnowledge the friendship, comradeship and support over many years of Darragh O'Donoghue, whose untimely death has robbed us all. He is sorely missed. 


\section{References}

[1] N. Law, et al., 2009, The Palomar Transient Factory: System Overview, Performance, and First Results, PASP, 121, 1395

[2] B. J. Shappee, et al., 2014, The Man behind the Curtain: X-Rays Drive the UV through NIR Variability in the 2013 Active Galactic Nucleus Outburst in NGC 2617, ApJ, 788, 48

[3] A. J. Drake, et al., 2009, First Results from the Catalina Real-Time Transient Survey, ApJ, 696, 870

[4] V. Lipunov, et al., 2010, MASTER Robotic Net, Advances in Astronomy, Volume 2010, Article ID 349171

[5] M. A. C. Perryman et al., 2001, GAIA: Composition, formation and evolution of the Galaxy, A\&A, 369,339

[6] See www.ast.uct.ac.za/meerlicht/

[7] K. W. Hodapp, et al., 2004, Optical design of the Pan-STARRS telescopes, Proc. SPIE, 5489, 667

[8] S. Keller, et al., 2007, The SkyMapper Southern Sky Survey, PASA, 24, 1

[9] Z. Ivezic et al., 2008, LSST: from Science Drivers to Reference Design and Anticipated Data Products, preprint (astro-ph/0805.2366)

[10] D.A.H. Buckley, 2013, Selecting, scheduling and carrying out observing programmes at SALT. In "Organizations, People and Strategies in Astronomy" - Volume 2 (OPSA 2), A. Heck (Ed), ISBN 978-2-9542677-1-5

[11] D. A. H. Buckley, et al., 2006, Status of the Southern African Large Telescope (SALT) FirstGeneration Instruments, Proc. SPIE 6269, 62690A

[12] P. Väisänen, 2015, How to best use SALT: maximizing your chances, proceeding of SALT Science Conference 2015, paper 7 these proceedings.

[13] M. Still et al., 2006, SALT spectroscopy of GRB 060605, GCN \#5226.

[14] R. Fesen, D. Milisavljevic, private communication.

[15] D.A.H. Buckley, et al., 2015, SALT spectral classification of MASTER OT J074858.45-655344.6.5 as a likely Dwarf Nova in outburst, ATel \#7165

[16] D.A.H. Buckley, et al., 2015, Classification of MASTER OT J061451.70-272535.5 as an eclipsing Polar, ATel \#7169

[17] D.A.H. Buckley, et al., 2015, SALT spectroscopy of the flaring blazar J141922.55-083832.0, ATel $\# 7167$

[18] V. G. Kornilov et al, 2012, Robotic optical telescopes global network MASTER II. Equipment, structure, algorithms, Exp Astron 33:173-196

[19] D. A. H. Buckley et al., 2014, GRB 141225A: MASTER-SAAO first OT detection, GCN \#17237

[20] D. A. H. Buckley et al., 2014, GRB 150301B: MASTER early OT detection, GCN \#17518

[21] E. S. Gorbovskoy, et al., 2015, Early polarization observations of the optical emission of gammaray bursts: GRB150301B and GRB150413A, MNRAS, in press. 